\title{
Variabilité géographique et adaptation aux contraintes du milieu méditerranéen des pins de la section halepensis : résultats (provisoires) d'un essai en plantations comparatives en France
}

\author{
M Bariteau
}

\author{
avec la collaboration technique de J Pommery, \\ INRA domaine du Ruscas, 83237 Bormes-les-Mimosas cedex
}

\begin{abstract}
INRA, station de génétique et d'amélioration des arbres forestiers avenue Vivaldi, 84000 Avignon. France
\end{abstract}

(Reçu le 30 janvier 1991; accepté le 11 février 1992)

\begin{abstract}
Résumé - Les résultats de plantations comparatives effectuées sur 2 sites (Vitrolles et Ceyreste) dans le Sud de la France dans le cadre d'un essai international sur les principales provenances de pins de la section halepensis sont présentés, 11 ans et 13 ans après la mise en place. Les caractères étudiés sont : la résistance au froid; la résistance à la sécheresse; la résistance à la chenille processionnaire (Thaumetopea pithyocampa); la croissance en hauteur. Pour Pinus halepensis, les résultats acquis à Vitrolles, où l'espèce est bien adaptée, permettent de confirmer la bonne adaptation de la provenance locale et l'intérêt de certaines provenances espagnoles et du Nord-Est de la Grèce. Pour Pinus brutia, les sites expérimentaux ne correspondent pas à l'aire potentielle d'introduction en France, située plus en altitude. Les sélections opérées par le gel et la sécheresse, ainsi que les connaissances déjà acquises dans l'aire naturelle, permettent néanmoins de proposer un choix de provenances pour la zone supra-méditérranéenne française (étage du chêne pubescent), en fonction des risques potentiels de sécheresse.

Les possibilittés d'hybridation entre Pinus brutia et Pinus halepensis ouvrent des voies d'études et de création variétale intéressantes mais imposent également des mesures de protection et de conservation de la ressource existante.
\end{abstract}

amélioration génétique / forêt méditerranéenne / Pinus halepensis / Pinus brutia / Pinus eldarica

Summary - Geographic variation and stress adaptation of Pinus halepensis - Pinus brutia complex in Mediterranean conditions. Preliminary results of a provenance test in France. Results of a 2-site provenance trial planted in south-eastern France (Vitrolles and Ceyreste) as part of an international network involving pine provenances of the Pinus halepensis - Pinus brutia complex are presented. Observations were made 11 and 13 years after plantation. Traits studied are frost and drought resistance, height growth and resistance to the processionary moth (Thaumetopea pithyocampa). For results from the Aleppo pine, the Vitrolles site, which is typical for this species, con-

* Correspondence and reprints 
firms that the local provenances, as well as those from Spain and north-eastern Greece show good site adaptation. Pinus brutia is not well adapted to either site due to its altitude, but a choice of provenances can be proposed for afforestation in the more elevated pubescent oak range, relative to their resistance to frost and drought, as well as their behaviour in natural stands. As the 2 species, ie Pinus brutia and Pinus halepensis hybridize easily, the future improvement programme may involve a hy brid breeding population; however, the protection and conservation of the local French Aleppo pine resources should also be considered.

tree breeding / Mediterranean forest / Pinus halepensis / Pinus brutia / Pinus eldarica

\section{INTROUUCTION}

Les pins de la section halepensis peuvent être scindés en 2 espèces : Pinus halepensis Miller et Pinus brutia Tenore (Nahal, 1962). La première se répartit sur le pourtour occidental de la Méditerranée, la seconde étant strictement orientale. Des formes géographiques distinctes existent au sein de Pinus brutia qui sont parfois décrites en tant qu'espèces à savoir : Pinus pithyusa Stevens, Pinus eldarica Hedwedjew et Pinus stankwiczii (Debazac, 1964). Une grande hétérogénéité a été notée par divers auteurs au sein des pins de la section halepensis. Debazac et Tomassone (1965) remarquent un gradient géographique : les dimensions et poids de graines, ainsi que le nombre et la longueur des cotylédons croissent dans le même sens d'ouest en est. Les pins d'Afrique du Nord et du Nord de la Méditerranée occidentale semblent distincts.

La virriabilité des pins de la section halepensis se traduit sur de nombreux caractères dont la forme. Arbez (1974) cite l'existence de peuplements de Pinus brutia ayant une forme "excellente" : cela permet de penser que l'espèce a un bel avenir pour le reboisement en zone méditerranéenne. Mais la forte variabilité géographique rend difficile le choix de l'origine des graines pour le reboisement, en particulier là où il n'y a pas de sources locales utilisables. Une expérience internationale de comparaison des provenances de Pinus halepensis et Pinus brutiaeldarica a été initiée en 1975 par la FAO (projet FAO/SCM/CRFM/4bis). Les objectifs et la méthodologie ont été présentés dans un protocole commun à tous les pays participants (Eccher, 1975).

Les premiers résultats traitent essentiellement des données de pépinière (Bellefontaine et Raggabi, 1977; Pelizzo et Tocci, 1978; Calamassi et al, 1980). Des observations, 10 ans après plantation, ont été publiées par Allemand et al (1985) sur l'expérimentation française, ainsi que par Eccher et al (1987) sur les essais effectués en Italie. Ces derniers mettent en évidence une différence d'accroissement entre pin d'Alep et pin brutia au stade juvénile au profit du pin d'Alep, qui s'atténue au cours du temps. Dans les stations chaudes et arides, c'est Pinus halepensis qui reste supérieur à Pinus brutia pour ce qui est des accroissements en hauteur. Les provenances de pin d'Alep de Grèce orientale (péninsule de Chalkidike et Eubée septentrionale) sont très performantes, tant en ce qui concerne la croissance que de la résistance au stress hydrique. Globalement, les provenances de Pinus halepensis sont plus résistantes à la sécheresse que celles de Pinus brutia, à l'exception des provenances montagnardes Isparta et Pamuçak: ces dernières se sont également révélées très résistantes au gel ainsi que les provenances de Pinus eldarica. Le port et la forme des fûts sont en général meilleurs pour Pinus brutia et Pinus eldarica que pour Pinus halepensis.

Les meilleurs génotypes testés en Israël, à partir de critères de croissance et survie sur des plants de 10 ans, sont des 
provenances locales ou celles issues de basse altitude en Grèce pour $P$ halepensis, et pour $P$ brutia, les provenances de basse altitude de la côte méditerranéenne turque: Bakara, Marmaris, Düzlerçani (Weinstein, 1989).

Le présent article se propose de faire le point sur l'expérimentation française, 12 ans après la première plantation en examinant les résultats sur la croissance en hauteur, la résistance au froid, à la sécheresse, et à la chenille processionnaire (Thaumetopea pithyocampa).

\section{MATÉRIEL ET MÉTHODES}

\section{Sites expérimentaux et matériel végétal}

Deux sites ont été retenus : Vitrolles et Ceyreste dans les Bouches-du-Rhône. Les tableaux I et II présentent de façon synthétique les principales caractéristiques et les résultats d'analyses chimiques des 2 sites.

L'hétérogénéité du site de Vitrolles a nécessité une étude complémentaire par le CEMAGREF d'Aix-en-Provence qui a distingué 3 zones, dénommées a posteriori comme "bonnes", "moyennes" ou "mauvaises" en fonction des résultats de croissances en hauteur et des survies, toutes provenances confondues.

Le tableau III présente l'échantillonnage des provenances testées en France au sein de la collection internationale (d'après Eccher et al, 1987). Les provenances sont localisées sur la figure 1 .

\section{Dispositifs expérimentaux}

Les caractéristiques des dispositifs sont les suivantes:

- Vitrolles : blocs incomplets à composition aléatoire; 55 blocs de 6 provenances par parcelle unitaire de 8 plants;

Tableau 1. Principales caractéristiques des dispositifs français.

Vitrolles Ceyreste

\section{Conditions écologiques}

Altitude
Longitude-latitude
Exposition/pente
Pluviométrie (1951/1980)
Sol
Végétation

Surface

Travail du sol

\section{Plantation \\ Date \\ Espacement \\ Age des plantes Méthode}

$200 \mathrm{~m}$
$3,26 \mathrm{gr} \mathrm{E} / 48,32 \mathrm{gr} \mathrm{N}$
Plateau/nulle
$569 \mathrm{~mm}$ (Marignane)
Calcaire
Forêt de Pinus halepensis
brûlée en 1972

1,93 ha

Trait de ripper tous les $5 \mathrm{~m}$

Mars 1978
$5 \times 1,5 \mathrm{~m}$ sur trait
$1-0$ fertil pot
Au potet dans trait
de ripper

$470 \mathrm{~m}$

$3,71 \mathrm{gr} \mathrm{E} / 48,4 \mathrm{gr} \mathrm{N}$

Sud $/ 10 \%$ $786 \mathrm{~mm}$ (Cuges)

Grès du Baguier Chênes verts et kermes - arbousier

1,13 ha

Sous-solage croisé en plein

Janvier 1976

$2,5 \times 1,33$

1-0 fertil pot

Au potet dans raie

de sous-solage 


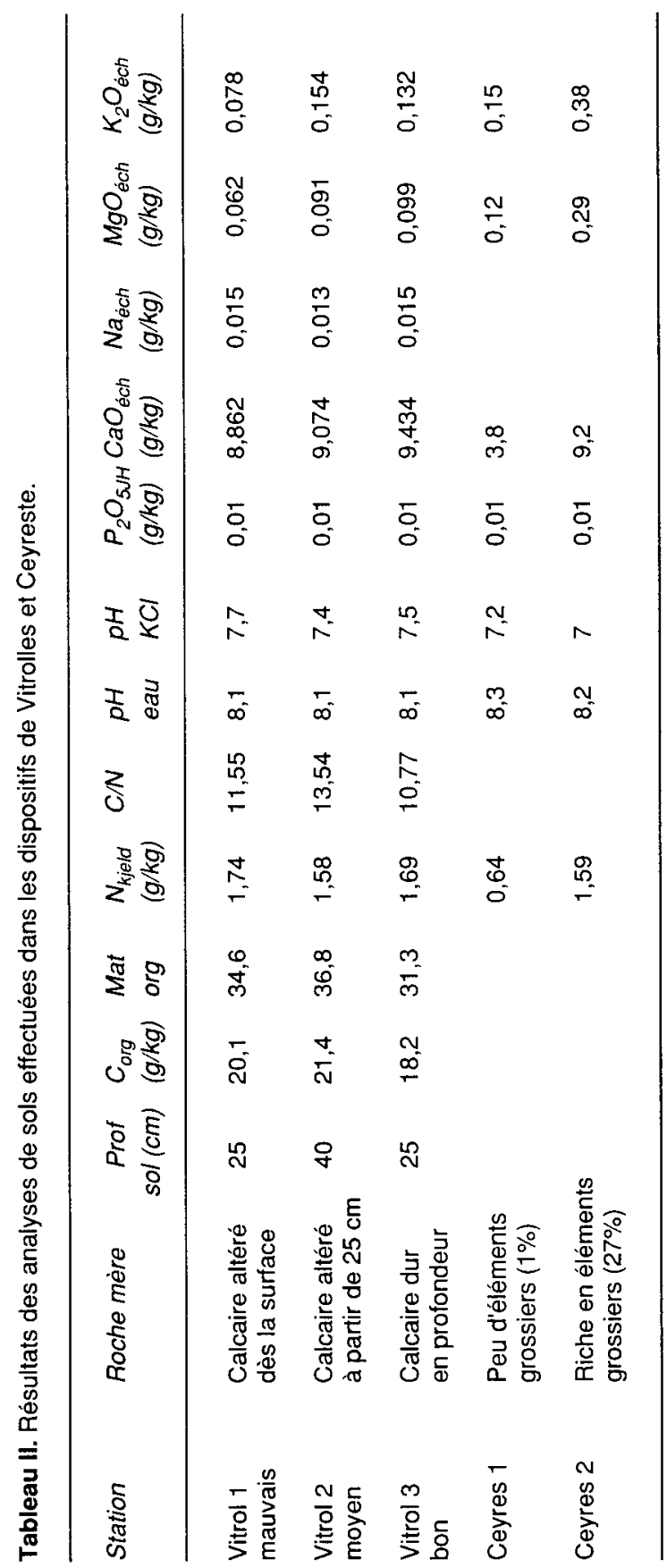


Tableau III. Liste des provenances utilisées dans l'essai international sur les pins de la section halepensis. Ind: indicatif internation ( $\mathrm{A}=$ Pinus halepensis; $\mathrm{B}=$ Pinus brutia; $\mathrm{E}=$ Pinus eldarica). Alt : altitude des peuplements (en $\mathrm{m}$ ); $N$ : numéro dans les essais français. Disp : utilisation des dispositions français $(V=$ Vitrolles; $C=$ Ceyreste $)$.

\begin{tabular}{|c|c|c|c|c|c|c|c|}
\hline Ind & Provenance & Pays & Longit & Latit & Alt & $N$ & Disp \\
\hline A1 & Albanie & Albanie & $19^{\circ} 25^{\prime} \mathrm{E}$ & $40^{\circ} 37^{\prime}$ & 2 & & \\
\hline A2 & Eléa & Grèce & $21^{\circ} 32^{\prime} \mathrm{E}$ & $37^{\circ} 46^{\prime}$ & 200 & 1 & $\mathrm{~V} / \mathrm{C}$ \\
\hline $\begin{array}{l}\text { A3 } \\
\text { A4 }\end{array}$ & $\begin{array}{l}\text { Euboea } \\
\text { Chalkidike I }\end{array}$ & $\begin{array}{l}\text { Grèce } \\
\text { Grèce }\end{array}$ & & $38^{\circ} 58^{\prime}$ & 200 & $?$ & \\
\hline A5 & Chalkidike II & $\begin{array}{l}\text { Grèce } \\
\text { Grèce }\end{array}$ & $\begin{array}{l}23^{\circ} 21^{\prime} \mathrm{E} \\
23^{\circ} 44^{\prime} \mathrm{E}\end{array}$ & $\begin{array}{l}40^{\circ} 1 f^{\prime} \\
40^{\circ} 03^{\prime}\end{array}$ & $\begin{array}{l}125 \\
70\end{array}$ & 2 & $\mathrm{~V} / \mathrm{C}$ \\
\hline A6 & Shaharia & Israë| & $34^{\circ} 50^{\prime} E$ & $31^{\circ} 36^{\prime}$ & 200 & & \\
\hline A7 & Elkosh & israël & $35^{\circ} 18^{\prime} \mathrm{E}$ & $33^{\circ} 01^{\prime}$ & 500 & & \\
\hline$A 8$ & Sakiet Sidiyoussef & Tunisie & $8^{\circ} 25^{\prime} E$ & $36^{\circ} 15$ & 700 & 3 & $\mathrm{~V} / \mathrm{C}$ \\
\hline $\begin{array}{l}\text { A9 } \\
\text { A10 }\end{array}$ & $\begin{array}{l}\text { Oum Djeddour } \\
\text { Diebel Selloum }\end{array}$ & $\begin{array}{l}\text { Tunisie } \\
\text { Tunisie }\end{array}$ & $\begin{array}{l}8^{\circ} 57^{\prime} \mathrm{E} \\
8^{\circ} 40^{\prime} \mathrm{E}\end{array}$ & $\begin{array}{l}35^{\circ} 38^{\prime} \\
35^{\circ} 05^{\prime}\end{array}$ & $\begin{array}{l}900 \\
900\end{array}$ & & \\
\hline A11 & Aures Tababuel & Algérie & $6^{\circ} 50^{\prime} \mathrm{E}$ & $35^{\circ} 10^{\prime}$ & & & \\
\hline A12 & Zaouia Ifrane & Maroc & $5^{\circ} 23^{\prime} \mathrm{E}$ & $33^{\circ} 15^{\prime}$ & 1250 & 4 & $\mathrm{~V} / \mathrm{C}$ \\
\hline A13 & J Afra Selminte & Maroc & $7^{\circ} 55^{\prime} \mathrm{W}$ & $30^{\circ} 44^{\prime}$ & 1600 & & \\
\hline $\begin{array}{l}\text { A } 14 \\
\text { A15 }\end{array}$ & Ouardane Bouksane & Maroc & $5^{\circ} 08^{\prime} \mathrm{W}$ & $35^{\circ} 03^{\prime}$ & 900 & 5 & $\mathrm{~V} / \mathrm{C}$ \\
\hline $\begin{array}{l}\text { A15 } \\
\text { A16 }\end{array}$ & Tamga Zaouia & Maroc & $6^{\circ} 07^{\prime} \mathrm{W}$ & $32^{\circ} 02^{\prime}$ & 1250 & & \\
\hline $\begin{array}{l}\text { A16 } \\
\text { A17 }\end{array}$ & $\begin{array}{l}\text { Soportujar } \\
\text { Guadalmedina }\end{array}$ & Espagne & $3^{\circ} 15^{\prime} \mathrm{W}$ & $37^{\circ} 10^{\prime}$ & 800 & 6 & $\mathrm{~V} / \mathrm{C}$ \\
\hline $\begin{array}{l}\text { A17 } \\
\text { A18 }\end{array}$ & $\begin{array}{l}\text { Guadalmedina } \\
\text { Maria }\end{array}$ & $\begin{array}{l}\text { Espagne } \\
\text { Espagne }\end{array}$ & $\begin{array}{l}2^{\circ} 15^{\prime} \mathrm{W} \\
2^{\circ} 10^{\prime} \mathrm{W}\end{array}$ & $37^{\circ} 02^{\prime}$ & 1200 & & \\
\hline A19 & Cehegin & Espagne & $1^{\circ} 55^{\prime} \mathrm{W}$ & $38^{\circ} 05^{\circ}$ & 850 & 7 & $\mathrm{~V} / \mathrm{C}$ \\
\hline $\mathrm{A} 20$ & Jarafuel & Espagne & $1^{\circ} 00^{\prime} \mathrm{W}$ & $38^{\circ} 55^{\circ}$ & 600 & & \\
\hline A21 & Serra & Espagne & $0^{\circ} 28^{\prime} \mathrm{W}$ & $39^{\circ} 50^{\prime}$ & 600 & 8 & $\mathrm{~V} / \mathrm{C}$ \\
\hline A22 & Montmell & Espagne & $1{ }^{\circ} 32 \mathrm{E}$ & $41^{\circ} 24^{\prime}$ & 400 & & \\
\hline A23 & Tarrasa & Espagne & $2^{\circ} 06^{\prime} \mathrm{E}$ & $41^{\circ} 28^{\prime}$ & 250 & 9 & $\mathrm{~V} / \mathrm{C}$ \\
\hline $\begin{array}{l}\text { A24 } \\
\text { A25 }\end{array}$ & Gemenos & $\begin{array}{l}\text { France } \\
\text { Italie. }\end{array}$ & $\begin{array}{l}5^{\circ} 40^{\prime} \mathrm{E} \\
8^{\circ} 03^{\prime} \mathrm{E}\end{array}$ & $43^{\circ} 25^{\prime}$ & 150 & 10 & $\mathrm{~V} / \mathrm{C}$ \\
\hline $\begin{array}{l}A 25 \\
A 26\end{array}$ & Otricoli & Italie & $12^{\circ} 38^{\prime} \mathrm{E}$ & $\begin{array}{l}43^{\circ} 34 \\
42^{\circ} 24^{\prime}\end{array}$ & $\begin{array}{l}200 \\
400\end{array}$ & 11 & $V / C$ \\
\hline A27 & Vico del Gargano & Italie & $16^{\circ} 00^{\prime} \mathrm{E}$ & $41^{\circ} 54^{\prime}$ & $\begin{array}{l}400 \\
225\end{array}$ & 12 & V/C \\
\hline A28 & Patemisco & Italie & $17^{\circ} 20^{\prime} \mathrm{E}$ & $40^{\circ} 39^{\prime}$ & & & \\
\hline A29 & Aures Beni Melloul & Algérie & $6^{\circ} 50^{\prime} \mathrm{E}$ & $35^{\circ} 10^{\prime}$ & 1700 & & \\
\hline $\begin{array}{l}\mathrm{A} 30 \\
\mathrm{~A} 31\end{array}$ & $\begin{array}{l}\text { Senalba } \\
\text { Telagh }\end{array}$ & $\begin{array}{l}\text { Algérie } \\
\text { Alqúrie }\end{array}$ & & & & & \\
\hline A32 & Ouarsenis & Algérie & $5^{\circ} 04^{\prime} \mathrm{E}$ & $35^{\circ} 05^{\prime}$ & & & \\
\hline A33 & Liban & Liban & & & & & \\
\hline B1 & Chania & Grèce & $23^{\circ} 57^{\prime} \mathrm{E}$ & $35^{\circ} 17^{\prime}$ & 850 & 13 & $\mathrm{~V} / \mathrm{C}$ \\
\hline $\mathrm{B} 2$ & Kavala & Grèce & $24^{\circ} 42^{\prime} \mathrm{E}$ & $40^{\circ} 48^{\prime}$ & 100 & & \\
\hline B3 & Lassithiou & Grèce & $25^{\circ} 32^{\prime} \mathrm{E}$ & $35^{\circ} 06^{\prime}$ & 1100 & 14 & $\mathrm{~V} / \mathrm{C}$ \\
\hline B4 & Alexandropolis & Grèce & $26^{\circ} 13^{\prime} \bar{E}$ & $41^{\circ} 08^{\prime}$ & 200 & 15 & $\mathrm{~V} / \mathrm{C}$ \\
\hline B5 & Chyptre & Chypre & $33^{\circ} 17^{\prime} \mathrm{E}$ & $35^{\circ} 08^{\prime}$ & 150 & & \\
\hline $\mathrm{B} 6$ & Marmaris & Turquie & $28^{\circ} 18^{\prime} \mathrm{E}$ & $37^{\circ} 00^{\prime}$ & 175 & 16 & $V / C$ \\
\hline B7 & Isparta & Turquie & $29^{\circ} 32^{\prime} \mathrm{E}$ & $38^{\circ} 04^{\prime}$ & 1043 & 17 & $\mathrm{~V} / \mathrm{C}$ \\
\hline B8 & Düzlerçani & Turquie & $30^{\circ} 25^{\prime} \mathrm{E}$ & $37^{\circ} 03^{\prime}$ & 250 & 18 & $\mathrm{~V} / \mathrm{C}$ \\
\hline B9 & Pamuçak & Turquie & $30^{\circ} 41^{\prime} \mathrm{E}$ & $37^{\circ} 40^{\prime}$ & 780 & 19 & $\mathrm{~V} / \mathrm{C}$ \\
\hline $\mathrm{B} 10$ & Bozburun & Turquie & $30^{\circ} 45^{\prime} \bar{E}$ & $37^{\circ} 21^{\prime}$ & 520 & 20 & $\mathrm{~V} / \mathrm{C}$ \\
\hline $\mathrm{B} 11$ & Bakara & Turquie & $32^{\circ} 43^{\prime} \mathrm{E}$ & $36^{\circ} 09^{\prime}$ & 300 & 21 & $\mathrm{~V} / \mathrm{C}$ \\
\hline $\mathrm{B} 12$ & Silifke & Turquie & $33^{\circ} 43^{\prime} \mathrm{E}$ & $36^{\circ} 13^{\prime}$ & 100 & 22 & $\mathrm{~V} / \mathrm{C}$ \\
\hline $\mathrm{B} 13$ & Camgölü & Turquie & $35^{\circ} 20^{\prime} \mathrm{E}$ & $41^{\circ} 50^{\prime}$ & 70 & 23 & $\mathrm{~V} / \mathrm{C}$ \\
\hline B14 & Baspinar & Turquie & $35^{\circ} 15^{\prime} E$ & $37^{\circ} 48^{\prime}$ & 700 & 25 & \\
\hline $\mathrm{B} 15$ & Kisildag & Turqie & $35^{\circ} 58^{\prime} \mathrm{E}$ & $36^{\circ} 21^{\prime}$ & 370 & 24 & $\mathrm{~V} / \mathrm{C}$ \\
\hline $\begin{array}{l}\text { B16 } \\
\text { B17 }\end{array}$ & Zawita & Irak & $44^{\circ} 20^{\prime} E$ & $36^{\circ} 35^{\prime}$ & & & \\
\hline $\mathrm{B} 1$ & $\begin{array}{l}\text { Liban } \\
\text { Iran I }\end{array}$ & Liban & & & & 26 & \\
\hline E2 & Iran II & $\begin{array}{l}\text { Iran } \\
\text { Iran }\end{array}$ & & & & 27 & $\breve{C}$ \\
\hline E3 & Iran III & Iran & & & & 28 & $\mathrm{C}$ \\
\hline
\end{tabular}


- Ceyreste : lattice équilibré : 25 provenances testées ( $P$ eldarica est en dispositif complémentaire sous forme de placettes surnuméraires de 20 plants dans chaque répétition complète); 5 provenances par bloc; 6 répétitions; 20 plants par parcelle unitaire (12 plants pour Gemenos).

\section{Méthodes d'analyse}

\section{Résistance au froid}

Elle a été testée à la suite d'une forte gelée en janvier 1985; les conséquences pouvant s'étaler sur plusieurs années, le taux de mortalité à terme par gel (sur une période de 3 ans suivant la gelèe) a été calculée pour chaque provenance (Mgelp) par comparaison des mortalités entre 1988 et 1985 . Les arbres notés indemnes après le gel de 1985 ont une survie de $99,3 \%$ en 1988 et on acceptera, par approximation, que la mortalité entre 1985 et 1988 est à imputer à l'action du froid de 1985.

\section{Résistance à la sécheresse}

Une notation individuelle a été effectuée à Vitrolles en janvier 1990 selon le barème suivant : $0=$ arbre indemne; 1 = arbre stressé (jaunissement, chute d'aiguilles); 2 = arbre mort en 1989; $-9=$ arbre mort avant 1989.

Le taux de survie, depuis la plantation, a été calculé pour chaque provenance (Surv90p), en prenant comme vivants les arbres notés 0 et 1 . II s'agit donc d'un bilan provisoire, par comparaison des survies en 1988 et en 1990, qui fait état d'une mortalité minimale (Msecp), pour le cas où tous les plants notés "douteux" survivraient: Msecp $=($ Surv88p-Surv90p) $/$ Surv88p

À Ceyreste, la mortalité liée à la sécheresse de l'année 1989 est faible et n'a pas justifié de notation.

Une analyse de variance a été effectuée sur les 24 provenances et les 3 zones (voir plus haut Sites expérimentaux) en prenant pour modèle :

$M s e c p z=$ effet provenance + effet zone + valeur résiduelle.

(Msecpz $=$ mortalité de la provenance $p$ dans la zone z).
Un test de Duncan au seuil de confiance de $5 \%$ permet de classer les provenances.

\section{Résistance à la chenille processionnaire}

Elle a été notée en présence - abscence pour chaque individu en mai 1984, à la suite d'une attaque importante sur le dispositif de Ceyreste. Le pourcentage d'attaques a été calculé pour chaque provenance (Pourp) et pour chaque répétition (Pourr). Les provenances sont classées en fonction de la gravité de l'attaque; d'autre part les différences de taux d'attaques suivant les répétitions sont testées à l'aide d'un $\chi^{2}$. La mortalité résultant de l'attaque de processionnaires n'a pu être évaluée étant donné l'intensité du gel l'hiver suivant.

\section{Croissances en hauteur}

Des mesures individuelles, au $\mathrm{cm}$ près, ont été réalisées sur la hauteur totale en 1988 (HT88) pour les 2 dispositifs. Les arbres morts au moment des mesures sont notés -9 , ce qui permet de calculer les taux de survie. Une analyse de variance est construite sur le modèle :

$$
H T_{i j k}=m+p_{i}+b_{j}+l_{i j}+e_{i j k} ;
$$

où $H T_{i j k}$ représente les valeurs individuelles de la variable $\mathrm{HT} 88$ et $p_{i}, b_{j}, l_{i j}$ sont respectivement les effets des facteurs provenances, blocs ainsi que l'effet d'interaction entre la provenance $i$ et le bloc $j ; e_{i j k}$ est la valeur résiduelle. Dans les 2 cas, les résultats des analyses montrent un effet d'interaction significatif au seuil de $1 \%$, vraisemblablement lié à la forte hétérogénéité du sol dans les 2 sites, y compris à l'intérieur d'un même bloc (tableau IV). Finalement, le dispositif de Vitrolles a été restructuré sur des critères d'homogénéité de hauteurs, de survie, et de disposition sur le terrain. Trois zones distinctes notées "médiocre" (1), "moyenne" (2) et "bonne" (3) ont été définies (voir tableau II) et un échantillon de 10 plants vivants en 1988 a été tiré au hasard pour chaque provenance et dans chaque zone. Quatre provenances possèdent des effectifs trop faibles pour être utilisées dans cette analyse (Elea, Gemenos, Alexandropolis, Bozburun). Les moyennes globales obtenues 
Tableau IV. Analyses de variances sur les hauteurs mesurées en 1988 à Vitrolles et à Ceyreste. Source : source de variation; ddl : nombre de degrés de liberté; ms: carré moyen; $F$ : valeur calculée pour le test $F\left({ }^{* * *}=\right.$ significatif au seuil de $\left.1 / 1000\right)$. Programme utilisé : module GLM type III bibliothèque SAS'STAT.

\begin{tabular}{|c|c|c|c|c|}
\hline Site & Source & $d d l$ & $m s$ & $F$ \\
\hline Vitrolles & $\begin{array}{l}\text { Provenance } \\
\text { Bloc } \\
\text { Interaction }\end{array}$ & $\begin{array}{r}23 \\
54 \\
238\end{array}$ & $\begin{array}{r}18895,47 \\
7976,26 \\
3001,55\end{array}$ & $\begin{array}{r}16,28^{\star \star \star *} \\
6,87^{\star * *} \\
2,59^{\star \star \star}\end{array}$ \\
\hline Ceyreste & $\begin{array}{l}\text { Provenance } \\
\text { Bloc } \\
\text { Interaction }\end{array}$ & $\begin{array}{r}26 \\
34 \\
100\end{array}$ & $\begin{array}{r}11342,35 \\
5838,87 \\
3269,92\end{array}$ & $\begin{array}{l}7,92^{\star \star \star \star} \\
4,07^{\star \star \star} \\
2,28^{* \star *}\end{array}$ \\
\hline
\end{tabular}

pour ces provenances sont calculées et comparées pour mémoire à celles de l'étude principale. Une analyse de variance est construite sur le modèle :

$$
Y_{i j k}=m+p_{i}+z_{j}+l_{i j}+e_{i j k} ;
$$

où $Y_{i j k}$ représente les valeurs individuelles de la variable étudiée (HT88); $p_{i}, z_{i}, l_{i j}$ sont respectivement les effets des facteurs provenances, zones ainsi que l'effet d'interaction entre la provenance $i$ et la zone $j ; e_{i j k}$ est la valeur résiduelle. Les moyennes sont comparées à l'aide d'un test de Newmann-Keuls au seuil de $5 \%$.

À Ceyreste, la mortalité très élevée et inégalement répartie sur le terrain, ainsi que les effets d'interaction déjà cités rendent délicate l'interprétation du lattice. II est préférable de considérer le dispositif comme un ensemble de 6 répétitions complètes. Pour tenir compte de l'hétérogénéité du terrain au sein des répétitions, une covariable "survie" est introduite au niveau de l'analyse. En effet, il existe une corrélation significative entre les hauteurs et les survies, ces 2 variables étant elles-mêmes liées à la fertilité des stations. Les pentes des corrélations établies provenance par provenance ne sont pas significativement différentes. La survie est calculée en 1988 sur chaque parcelle unitaire (Surv).

Les valeurs étudiées dans l'analyse sont des moyennes par provenance sur chaque parcelle unitaire (une parcelle unitaire par provenance dans chaque répétition). Une analyse de variance avec covariable est construite sur le modèle :

$$
Y_{i j}=m+p_{i}+a\left(\operatorname{Surv}_{i j}-m s\right)+e_{i j} ;
$$

où $Y_{i j}$ représente les valeurs individuelles pour la variable étudiée (HT88), $p_{i}$ l'effet du facteur provenance, Survij la survie de la provenance $i$ dans la répétition $j$, $m s$ la moyenne générale de la survie, eij le terme résiduel; $a$ est le coefficient de régression des valeurs Surv ${ }_{i j}$ sur $Y_{i j}$ La répétition 3 est éliminée de l'analyse, 25 parcelles sur 28 ayant totalement disparu. Une comparaison multiple de moyennes est effectuée à partir d'un test de Newman-Keuls au seuil de $5 \%$.

\section{RÉSULTATS}

\section{Résistance au froid}

La mortalité par gel est plus forte à Vitrolles qu'à Ceyreste : respectivement $15,7 \%$ et $9,4 \%$ (tableau V).

Les pins brutia et eldarica ont subi une mortalité toujours inférieure à $7 \%(2,7 \%$ en moyenne sur les 2 dispositifs pour Pinus brutia et $4,05 \%$ pour Pinus eldarica). Le 
Tableau V. Mortalités par gel. Ind : indicatif international. Prov : nom abrégé de la provenance; Gelv: mortalité à Vitrolles (\%); Gelc : mortalité à Ceyreste (\%); $M$ : mortalité moyenne sur les 2 dispositifs; $D$ : écart de mortalité entre les deux dispositifs (Gelv-Gelc); EFV : effectifs à Vitrolles (plants vivants avant le gel); $E F C=$ effectifs à Ceyreste (plants vivants avant le gel).

\begin{tabular}{|c|c|c|c|c|c|c|c|c|}
\hline Ind & Espèce & Prov & Gelv & Gelc & $M$ & $D$ & EFV & EFC \\
\hline $\mathrm{A} 24$ & $P$ halepensis & Geme & 0,0 & 0,0 & 0,0 & 0,0 & 16 & 24 \\
\hline B7 & $P$ brutia & Ispa & 0,9 & 0,0 & 0,45 & 0,9 & 115 & 69 \\
\hline B15 & P brutia & Kizi & 0,0 & 1,2 & 0,6 & $-1,2$ & 73 & 84 \\
\hline B9 & P brutia & Pamu & 1,9 & 0,0 & 0,95 & 1,9 & 103 & 73 \\
\hline B11 & $P$ brutia & Baka & 0,0 & 3,9 & 1,95 & $-3,9$ & 81 & 76 \\
\hline$B 13$ & P brutia & Camg & 2,9 & 1,4 & 2,15 & 1,5 & 136 & 72 \\
\hline $\mathrm{B} 1$ & P brutia & Chan & 2,4 & 2,0 & 2,2 & 0,4 & 83 & 50 \\
\hline $\mathrm{B} 12$ & P brutia & Slli & 0,7 & 3,8 & 2,25 & $-3,1$ & 151 & 79 \\
\hline B3 & P brutia & Lass & 4,7 & 0,0 & 2,35 & 4,7 & 86 & 49 \\
\hline B6 & P brutia & Marm & 4,2 & 1,5 & 2,85 & 2,7 & 72 & 65 \\
\hline $\mathrm{B} 10$ & P brutia & Bozb & 3,8 & 5,6 & 4,7 & $-1,8$ & 52 & 89 \\
\hline B4 & P brutia & Alex & 6,4 & 4,6 & 5,5 & 1,8 & 47 & 65 \\
\hline B8 & P brutia & Duzl & 6,7 & 5,9 & 6,3 & 0,8 & 134 & 34 \\
\hline A19 & $P$ halepensis & Cehe & 8,8 & 4,4 & 6,6 & 4,4 & 80 & 45 \\
\hline A21 & $P$ halepensis & Serr & 18,1 & 11,8 & 14,95 & 6,3 & 116 & 51 \\
\hline $\mathrm{A} 4$ & $P$ halepensis & Chal & 18,6 & 17,6 & 18,1 & 1,0 & 86 & 51 \\
\hline A16 & $P$ halepensis & Sopo & 30,4 & 7,0 & 18,7 & 23,4 & 115 & 71 \\
\hline $\mathrm{A} 12$ & $P$ halepensis & Ifra & 24,8 & 18,6 & 21,7 & 6,2 & 129 & 70 \\
\hline A23 & $P$ halepensis & Tara & 19,4 & 25,0 & 22,2 & $-5,6$ & 129 & 36 \\
\hline A26 & $P$ halepensis & Otri & 21,3 & 25,8 & 23,6 & $-4,5$ & 108 & 62 \\
\hline A27 & $P$ halepensis & Viga & 28,0 & 21,7 & 24,9 & 6,3 & 100 & 23 \\
\hline$A 8$ & $P$ halepensis & Syou & 38,9 & 22,2 & 30,6 & 16,7 & 108 & 54 \\
\hline A14 & $P$ halepensis & Bouk & 47,8 & 26,8 & 37,3 & 21,0 & 115 & 56 \\
\hline $\mathrm{A} 2$ & $P$ halepensis & Elea & 85,4 & 56,0 & 70,7 & 29,4 & 82 & 25 \\
\hline B14 & Pbrutia & Basp & & 3,6 & & & & 84 \\
\hline $\mathrm{E}_{1}$ & $P$ eldarica & Ira1 & & 5,0 & & & & 80 \\
\hline E2 & Peldarica & Ira2 & & 1,3 & & & & 80 \\
\hline E3 & $P$ eldarica & Ira3 & & 5,9 & & & & 68 \\
\hline
\end{tabular}

groupe des pins d'Alep apparait dans les 2 cas, plus sensible au gel que celui des pins brutia (en moyenne $24,10 \%$ ) sauf pour Gemenos (provenance française).

Les pins brutia d'altitude sont peu sensibles au froid (Pamuçak, Isparta, Chania, Lassithiou). La provenance turque Kizildag est également très résistante : les peuplements d'origine sont situés à $370 \mathrm{~m}$ d'altitude dans la zone méditerranéenne extrême orientale, sur les pentes de l'Amanos. Les provenances Bakarı, Cam- gölü (région de la mer Noire) et Silifke, bien que venant de zones d'altitude faible, sont également peu sensibles au froid. Par contre, les populations situées entre Marmaris et Antalya, c'est-à-dire sur la partie occidentale de la côte méditerranéenne turque, semblent, à altitudes égales, plus sensibles au gel que celles de la partie orientale : la provenance Bozburun, par exemple, est plus sensible que la plupart des pins brutia bien que venant d'une altitude moyenne $(500 \mathrm{~m})$. 
Parmi les provenances de Pinus halepensis, les populations d'Afrique du Nord et d'Italie sont particulièrement sensibles au froid, ainsi que la provenance grecque Elea (Péloponnèse) : les mortalités sont très élevées à Vitrolles pour 2 provenances d'Afrique du Nord (Ouardane Bouksane et Sakiet Sidi Youssef), une provenance espagnole (Soportujar) et la population Elea. L'effet du gel a été accentué sur ces provenances en raison de la faible hauteur des plants (gradient de froid ve's le sol). La provenance Elea doit vraisemblablement sa grande sensibilité au froid à son origine thermoméditerranéenne (peuplements à $200 \mathrm{~m}$ d'altitude en Grèce méridionale).

\section{Résistance à la sécheresse}

Par rapport à la moyenne calculée sur 30 ans (soit $569 \mathrm{~mm}$ ), le déficit pluviométrique à Marignane, station météorologique proche de Vitrolles, est de $156 \mathrm{~mm}$ en 1988 , et $369 \mathrm{~mm}$ en 1989 (pluviométrie de l'année : $200 \mathrm{~mm}$ ). L'analyse de variance faite sur la mortalité par sécheresse à Vitrolles en 1990 montre un effet provenance et un effet zone significatifs (respectivement $F=2,80$, significatif au seuil de $1 \%$ et $F=6,67$, significatif au seuil de $1 \%$ : tableau VI). La zone 1 (médiocre) est distincte des 2 autres : la mortalité par sécheresse y est près de 2 fois supérieure à celle observée ailleurs. Une charge en

Tableau VI. Mortalité par sécheresse à Vitrolles en 1989. Ind : indicatif international. MSECp : mortalité moyenne par provenance (\%); $M S E C z$ : mortalité par zone (\%). Les traits verticaux relient les provenances ou les zones non significativement différentes d'après le test de Duncan au seuil de $5 \%$.

\begin{tabular}{|c|c|c|c|c|c|}
\hline Ind & Espèce & Provenance & MSECp & Zone & $\mathrm{MSECz}$ \\
\hline $\begin{array}{l}\text { B4 } \\
\text { B15 } \\
\text { A19 } \\
\text { A21 } \\
\text { A4 } \\
\text { A24 } \\
\text { A16 } \\
\text { A26 } \\
\text { A23 } \\
\text { B6 } \\
\text { B10 } \\
\text { B12 } \\
\text { A8 } \\
\text { B3 } \\
\text { B7 } \\
\text { B8 } \\
\text { A14 } \\
\text { A2 } \\
\text { A12 } \\
\text { B13 } \\
\text { B9 } \\
\text { B1 } \\
\text { B11 } \\
\text { A27 }\end{array}$ & $\begin{array}{l}\text { P brutia } \\
\text { P brutia } \\
\text { P halepensis } \\
\text { P halepensis } \\
\text { P halepensis } \\
\text { P halepensis } \\
\text { P halepensis } \\
\text { P halepensis } \\
\text { P halepensis } \\
\text { P brutia } \\
\text { P brutia } \\
\text { P brutia } \\
\text { P halepensis } \\
\text { P brutia } \\
\text { P brutia } \\
\text { P brutia } \\
\text { P halepensis } \\
\text { P halepensis } \\
\text { P halepensis } \\
\text { P brutia } \\
\text { P brutia } \\
\text { P brutia } \\
\text { P brutia } \\
\text { P halepensis }\end{array}$ & \begin{tabular}{l|} 
Alex \\
Kisi \\
Cehe \\
Serr \\
Chal \\
Geme \\
Sopo \\
Otri \\
Tara \\
Marm \\
Bozb \\
Sili \\
Syou \\
Lass \\
Ispa \\
Düzl \\
Bouk \\
Elea \\
Ifra \\
Camg \\
Pamu \\
Chan \\
Baka \\
Viga
\end{tabular} & $\begin{array}{r}5,46 \\
5,98 \\
7,11 \\
8,24 \\
9,72 \\
10,52 \\
11,45 \\
11,75 \\
12,55 \\
12,60 \\
14,20 \\
14,88 \\
15,78 \\
16,06 \\
17,60 \\
18,55 \\
18,65 \\
21,74 \\
21,76 \\
22,16 \\
24,65 \\
31,60 \\
32,64 \\
47,42\end{array}$ & $\begin{array}{ll}1 & 1 \\
3 & \\
2 & \end{array} \mid$ & $\begin{array}{l}24,09 \\
14,81 \\
13,54\end{array}$ \\
\hline
\end{tabular}


cailloux très importante dans les horizons de surface en est sans doute responsable (réserve en eau faible). Le classement des provenances en fonction de Msecp ne sépare pas Pinus halepensis et Pinus brutia (tableau VI) : l'effet espèce n'est pas significatif au seuil de $5 \%(F=0,19)$. Les 10 meilleures provenances sont cependant représentées par 7 provenances de pin d'Alep.

Contrairement à ce qui est constaté pour le pin brutia, sensibilité au gel et à la sécheresse vont de pair pour les pins d'Alep : les provenances d'Afrique du Nord ainsi que la provenance grecque Elea cumulent de mauvais résultats face à ces 2 formes de stress. La provenance italienne, Vico Del Gargano est une provenance mal adaptée aux caractéristiques écologiques du site de Vitrolles, ce qui s'est traduit par une très forte mortalité en 1989. Les provenances de Pinus halepensis Gemenos, Cehegin et dans une moindre mesure, Serra et Chalkidike sont peu sensibles au gel et à la sécheresse. Une seule provenance de pin brutia, Kisildag, cumule la résistance au gel et à la sécheresse.

\section{Résistance à la chenille processionnaire}

Le classement des provenances en fonction du pourcentage d'attaque est présenté dans le tableau VII. Le test de $\chi^{2}$ montre que les différences sont globalement significatives $\left(\chi^{2}=127,78\right.$ et ddl $=27$, significatif à $1 \%$ ). Le pourcentage le plus faible est obtenu pour un pin brutia de Crète (Chania), le plus fort pour un autre $P$ brutia grec (Alexandropolis) et une provenance de $P$ eldarica (Iran II). Pinus brutia-eldarica est plus sensible que Pinus halepensis : $93,25 \%$ d'attaque en moyenne contre $85,89 \%$. Les taux sont cependant élevés pour toutes les provenances. Nous n'avons pas trouvé de corrélations géographiques et écologiques (longitude, latitude, altitude) avec la sensibilité des provenances. Par contre, 2 paramètres expliquent bien les attaques:

- la position au sein de l'essai : certaines zones ont été plus particulièrement touchées (lisière sud du dispositif : répétitions 1 et 5);

- la hauteur des arbres au moment de l'attaque (HT83p mesurée en 1983), les

Tableau VII. Attaque de chenilles processionnaires dans le dispositif de Ceyreste en 1984. Ind : indicatif international. Pourp : pourcentage d'attaque (\%).

Ind Espèce Provenance Pourp Effectif

$\begin{array}{lllll}\text { B1 } & \text { P brutia } & \text { Chan } & 67,39 & 46 \\ \text { A14 } & \text { P halepensis } & \text { Bouk } & 70,91 & 55 \\ \text { A21 } & \text { P halepensis } & \text { Serr } & 76,47 & 51 \\ \text { A24 } & \text { P halepensis } & \text { Geme } & 79,17 & 24 \\ \text { A2 } & \text { P halepensis } & \text { Elea } & 80,00 & 25 \\ \text { A8 } & \text { P halepensis } & \text { Syou } & 83,02 & 53 \\ \text { B3 } & \text { P brutia } & \text { Lass } & 83,67 & 49 \\ \text { B12 } & \text { P brutia } & \text { Sili } & 85,90 & 78 \\ \text { A26 } & \text { P halepensis } & \text { Otri } & 86,44 & 59 \\ \text { A12 } & \text { P halepensis } & \text { Ifra } & 86,96 & 69 \\ \text { B8 } & \text { P brutia } & \text { Düzl } & 88,24 & 34 \\ \text { A19 } & \text { P halepensis } & \text { Cehe } & 91,11 & 45 \\ \text { B13 } & \text { P brutia } & \text { Camg } & 91,55 & 71 \\ \text { A4 } & \text { P halepensis } & \text { Chal } & 92,00 & 50 \\ \text { B6 } & \text { P brutia } & \text { Marm } & 92,31 & 65 \\ \text { B7 } & \text { P brutia } & \text { Ispa } & 92,54 & 67 \\ \text { E3 } & \text { P eldarica } & \text { Iran } & 92,54 & 67 \\ \text { B15 } & \text { P brutia } & \text { Kisi } & 93,75 & 80 \\ \text { A23 } & \text { P halepensis } & \text { Tara } & 94,29 & 35 \\ \text { A16 } & \text { P halepensis } & \text { Sopo } & 94,37 & 71 \\ \text { A27 } & \text { P halepensis } & \text { Viga } & 95,65 & 23 \\ \text { B9 } & \text { P brutia } & \text { Pamu } & 95,89 & 73 \\ \text { B10 } & \text { P brutia } & \text { Bozb } & 96,55 & 87 \\ \text { B14 } & \text { P brutia } & \text { Basp } & 97,56 & 82 \\ \text { B11 } & \text { P brutia } & \text { Baka } & 98,67 & 75 \\ \text { E1 } & \text { P eldarica } & \text { Iran1 } & 98,72 & 78 \\ \text { B4 } & \text { P brutia } & \text { Alex } & 100,00 & 65 \\ \text { E2 } & \text { P eldarica } & \text { Iran2 } & 100,00 & 80 \\ & & & & \end{array}$


grands sujets étant plus attaqués : une corrélation significative lie Pourp et HT83p (coefficient de corrélation 0,727 significatif à $1 \%$ ).

Les 2 provenances originaires de Crète (Chania et Lassithiou) se distinguent de l'ensemble des pins brutia par une hauteur faible en 1983 et un taux d'attaque nettement moindre. Les 2 valeurs restent en cohérence avec la relation liant Pourp et HT83p. L'origine de la sensibilité à la processionnaire ne semble donc pas être d'origine génétique mais il faudrait pouvoir en juger sur un dispositif approprié.

Certains auteurs confirment un déterminisme de l'attaque en fonction de la "silhouette" et du diamètre des aiguilles (Demolin, 1969). À Ceyreste, ce sont les cimes émergentes qui ont recueilli le plus de ponte, la variabilité de la morphologie des aiguilles entre provenances étant faible.

\section{Croissance en hauteur}

Le tableau VIII résume les principaux résultats. La moyenne globale est de 154,36 $\mathrm{cm}$ à Vitrolles, 11 ans après la plantation, et $143,97 \mathrm{~cm}$ à Ceyreste, 13 ans après la plantation. La meilleure provenance à $\mathrm{Vi}$ trolles (Cehegin) réalise un gain en hauteur de $20,7 \%$ par rapport à la moyenne. Ce gain est de $20,8 \%$ à Ceyreste pour la provenance de pin brutia, Marmaris.

Les pins d'Alep dominent les pins brutia à Vitrolles. Le phénomène inverse se produit à Ceyreste. Les provenances de Pinus eldarica occupent également la partie supérieure du classement sur ce site. Plusieurs constatations permettent d'expliquer cette forte interaction génotype $x$ environnement au niveau des espèces :

- à la suite d'une gelée sévère au cours de I'hiver 1976, les pins d'Alep ont subi une forte mortalité à Ceyreste, dès la plantation. Les mortalités comptées en 1983 en font bien état : $59,3 \%$ pour le pin d'Alep à Ceyreste contre $12,8 \%$ à Vitrolles; $41,8 \%$ pour le pin brutia à Ceyreste contre $8,5 \%$ à Vitrolles;

- les analyses de sol font apparaître peu de différences entre Vitrolles et Ceyreste, alors que la teneur en calcaire actif est vraisemblablement différente entre les 2 stations : traces pulvérulentes dans le sol et flore typiquement calcicole à Vitrolles (Erica multiflora), roche mère gréseuse et présence d'arbousiers (Arbutus unedo) à Ceyreste.

II n'a pas été trouvé de relations entre la croissance et l'origine géographique. Le poids des graines ou la taille des plants à 1 an ne sont pas indicateurs des performances analysées en 1988.

\section{DISCUSSION}

Le tableau IX fait la synthèse des résultats obtenus. Les provenances sont classées par ordre de rang moyen croissant (rang du classement sur les hauteurs en 1988). Les provenances de Pinus eldarica n'ont été plantées qu'à Ceyreste où les performances sont moyennes à bonnes. Cette espèce, testée en arboretums d'élimination ne donne de bons résultats que sur calcaire (Allemand, 1989). D'après des observations faites en plantation, elle semble par contre très sensible à de nombreux déprédateurs.

Les 3 provenances arrivant en tête de classement cumulent la résistance aux stress et des croissances bonnes sur les 2 sites d'expérimentation : Alexandropolis ( $P$ brutia), Chalkidike ( $P$ halepensis), Silifke ( $P$ brutia). D'une façon générale, les provenances les moins performantes sont aussi les plus sensibles au gel et à la sé- 


\begin{tabular}{|c|c|c|c|}
\hline Ind & Espèce & Provenance & HT88 \\
\hline \multicolumn{4}{|c|}{ Vitrolles } \\
\hline A19 & $P$ halep & Cehe & 186,33 \\
\hline A21 & $P$ halep & Serr & 185,67 \\
\hline A23 & $P$ halep & Tara & 179,83 \\
\hline A4 & $P$ halep & Chal & 174,67 \\
\hline $\mathrm{B} 12$ & $P$ brutia & Sili & 169,50 \\
\hline A27 & $P$ halep & Viga & 163,33 \\
\hline A12 & P halep & Ifra & 158,50 \\
\hline A16 & $P$ halep & Sopo & 156,55 \\
\hline$A B$ & $P$ halep & Syou & 155,50 \\
\hline B13 & Pbrutia & Camg & 152,50 \\
\hline A14 & $P$ halep & Bouk & 152,00 \\
\hline B7 & $P$ brutia & Ispa & 151,33 \\
\hline B9 & P brutia & Pamu & 150,00 \\
\hline A26 & $P$ halep & Otri & 147,33 \\
\hline Bt & P brutia & Chan & 147,33 \\
\hline B6 & Pbrutia & Marm & 146,33 \\
\hline B15 & Pbrutia & Kisi & 145,67 \\
\hline B11 & Pbrutia & Baka & 124,00 \\
\hline B8 & Pbrutia & Düzl & 123,17 \\
\hline B3 & $P$ brutia & Lass & 117,83 \\
\hline B4 & Pbrutia & Alex & 179,64 \\
\hline A24 & $P$ halep & Geme & 174,29 \\
\hline B10 & Pbrutia & Bozb & 143,12 \\
\hline$A 21$ & $P$ halep & Elea & 138,89 \\
\hline
\end{tabular}

Tableau VIII. Classement des provenances d'après les hauteurs totales en 1988 à Vitrolles et à Ceyreste. Les traits verticaux relient les provenances non significativement différentes dans le test de Newman-Keuls au seuil de $5 \%$.

cheresse. Cela tend à prouver que la croissance en hauteur est un caractère à déterminisme complexe et que dans le cas présent, il traduit bien l'adaptation globale du génotype au milieu. Dans une ambiance de sélection moins sévère, le classement aurait pu être sensiblement modifié. Cela pourrait expliquer, par action cumulée du gel puis de la sécheresse, certains résultats différents de ceux obtenus dans les essais italiens (Eccher et al, 1987) : Soportujar, Serra et Otricoli sont décrites par ces auteurs comme sensibles à la sécheresse, alors qu'elles figurent parmi les meilleures à Vitrolles $(8-12 \%$ de mortalité). Par contre, Vico del Gargano est également signalée comme sensible, ce qui est confirmé sur la dispositif français, la plus forte mortalité étant obtenue pour cette provenance $(47,5 \%)$. Pamuçak est décrite par Eccher comme résistante à la sécheresse alors qu'elle est parmi les provenances de $P$ brutia des plus sensibles à Vitrolles ( $24,7 \%$ de mortalité).

Les 2 meilleures provenances sont originaires du Nord-Est de la Grèce : Chalkidike est un pin d'Alep; Alexandropolis, un pin brutia. Quezel et Barbero (1985) notent que des phénomènes d'introgression sont possibles dans les zones de contact entre les 2 espèces. Panetsos (1986) a démontré l'intérêt des hybrides $P$ halepensis $\times P$ brutia. L'obtention est aisée en utilisant $P$ 
Tableau IX. Classement des provenances d'après le rang moyen sur les hauteurs en 1988.

\begin{tabular}{|c|c|c|c|c|c|c|c|c|}
\hline Espèce & Ind & Prov & $\mathrm{RV}$ & $\mathrm{RC}$ & $\mathrm{RM}$ & $\mathrm{Gel}$ & Séche & Forme \\
\hline P brutia & B14 & Basp & - & 4 & - & + & - & Accept \\
\hline P eldarica & E1 & Ira1 & - & 15 & - & + & - & * \\
\hline P eldarica & E2 & Ira2 & - & 3 & - & + & - & * \\
\hline P eldarica & E3 & Ira3 & - & 9 & - & + & - & * \\
\hline Pbrutia & B4 & Alex & 4 & 5 & 4,5 & + & + & * \\
\hline$P$ halepensis & A4 & Chal & 5 & 8 & 6,5 & $=$ & + & * \\
\hline Pbrutia & B12 & Sili & 7 & 7 & 7,0 & + & $=$ & Accept \\
\hline$P$ halepensis & A19 & Cehe & 1 & 16 & 8,5 & + & + & * \\
\hline Pbrutia & B6 & Marm & 18 & 1 & 9,5 & + & $=$ & Bon \\
\hline P brutia & B15 & Kisi & 19 & 2 & 10,5 & + & + & Très $B$ \\
\hline$P$ halepensis & A21 & Serr & 2 & 19 & 10,5 & $=$ & + & $*$ \\
\hline$P$ halepensis & $\mathrm{A} 23$ & Tara & 3 & 21 & 12,0 & - & $=$ & * \\
\hline P brutia & B7 & Ispa & 14 & 11 & 12,5 & + & $=$ & Accept \\
\hline P brutia & B13 & Camg & 12 & 14 & 13,0 & + & - & Accept \\
\hline P brutia & B9 & Pamu & 15 & 12 & 13,5 & + & - & Très B \\
\hline$P$ brutia & B11 & Baka & 22 & 6 & 14,0 & + & - & Except \\
\hline$P$ halepensis & A12 & Ifra & 9 & 20 & 14,5 & - & - & * \\
\hline P brutia & B10 & Bozb & 20 & 10 & 15,0 & + & $=$ & Bon \\
\hline$P$ halepensis & A24 & Geme & 6 & 24 & 15,0 & + & $=$ & * \\
\hline$P$ halepensis & A16 & Sopo & 10 & 22 & 16,0 & $=$ & $=$ & * \\
\hline P brutia & B1 & Chan & 16 & 17 & 16,5 & + & - & * \\
\hline$P$ halepensis & A27 & Viga & 8 & 26 & 17,0 & - & - & * \\
\hline P brutia & B3 & Lass & 24 & 13 & 18,5 & + & $=$ & * \\
\hline$P$ halepensis & $\mathrm{A} 8$ & Syou & 11 & 28 & 19,5 & - & $=$ & * \\
\hline$P$ halepensis & A14 & Bouk & 13 & 27 & 20,0 & - & $=$ & * \\
\hline$P$ brutia & $B 8$ & Duzl & 23 & 18 & 20,5 & + & $=$ & Accept \\
\hline$P$ halepensis & A26 & Otri & 17 & 25 & 21,0 & - & $=$ & * \\
\hline$P$ halepensis & $\mathrm{A} 2$ & Eléa & 21 & 23 & 22,0 & - & - & * \\
\hline
\end{tabular}

$\mathrm{RV}=$ rang obtenu sur le classement en hauteur 1988 à Vitrolles; RC = rang obtenu sur le classement en hauteur 1988 à Ceyreste; $\mathrm{RM}=$ rang moyen; Gel = mortalité moyenne par gel $(+)$ inférieure ou égale à $10 \% ;(=)$ supérieure à $10 \%$ et inférieure ou égale à $20 \% ;(-)$ supérieure à $20 \%$. Séche $=$ mortalité par sécheresse à Vitrolles (mêmes notations que pour Gel). Forme : notation effectuée dans l'air naturelle (Arbez, 1974). Accept = acceptable; Bon = bonne; Très $\mathrm{B}=$ très bonne; Except = exceptionnelle; ${ }^{*}$ : non décrite.

brutia comme femelle. Si les provenances issues des zones de contact des 2 espèces se révèlent performantes dans l'ensemble des essais internationaux, cela justifierait une intensification des recherches à leur sujet. Des peuplements naturels de pins d'Alep ont été décrits récemment en Turquie sur la côte Egéenne, entre Izmir et Marmaris, en contact direct avec des forêts de pins brutia (Alptekin,
1990). Une situation de même type existe dans la région d'Adana (Quezel, 1980).

Des recommandations en matière de choix de provenances en France peuvent être déduites des essais de Vitrolles et Ceyreste. La discussion doit tenir compte de 3 constatations préalables :

- les sites utilisés sont très limitants sur le plan de la fertilité (sécheresse; sol superfi- 
ciel); le terrain de Ceyreste est un milieu peu représentatif de zones potentielles de reboisement en France méditerranéenne;

- des études récentes sur les boisements français de pin brutia montrent que cette espèce devrait être utilisée dans une zone écologiquement différente de l'aire actuelle du pin d'Alep (Nouals et Boisseau, 1991) : climat méditerranéen atténué (étage supra-méditerranéen), notamment sur roches marneuses et substrats acides;

- étant donné les tempéraments très différents des espèces de pins de la section halepensis et les facilités d'hybridation naturelle, il serait bon de prendre des mesures d'inventaire et de conservation des plus beaux peuplements français de pins d'Alep, en particulier pour éviter la pollution génétique. Le pin brutia ne devrait pas être introduit à leur proximité.

Finalement l'essai de Vitrolles, qui est représentatif des conditions de sélection régnant dans l'air actuelle de Pinus halepensis en France (zone mésoméditerranéenne sur calcaire), permet d'apporter quelques conclusions sures pour cette espèce. Les provenances les mieux adaptées et dont la croissance est la moins faible sont représentées par 2 populations espagnoles (Cehegin, Serra), une population grecque (Chalkidike) et la provenance locale Gemenos. La législation actuelle prévoit pour le pin d'Alep l'utilisation obligatoire des provenances françaises. Des dérogations pourraient donc être accordées, les années où les peuplements autochtones ont une production insuffisante, pour les peuplements grecs et espagnols qui ont démontré leur adaptation à Vitrolles.

En matière de pin brutia, des plantations productives pourraient être envisagées dans les zones où le froid limite l'extension du pin d'Alep, à des altitudes moyennes où le cèdre de l'Atlas et les pins noirs ne sont pas encore à leur optimum écologique (étage inférieur du chêne pubescent). Les essais menés en France ne permettent pas d'extrapoler les résultats à ces sites de type "supra-méditerranéen". Les conclusions apportées en matière de résistance au stress hydrique et au froid, ainsi que les connaissances générales existantes sur l'espèce sont cependant suffisantes pour dégager quelques recommandations (en particulier, la variabilité des formes dans l'aire naturelle qui serait sous contrôle génétique; Arbez, 1974). Dans les zones supra-méditerranéennes, où existe un risque de sécheresse prolongée, d'origine édaphique ou climatique, 3 provenances peuvent être conseillées : Silifke, Marmaris et Kizildag. Ces provenances correspondent à des stations de faible altitude $(100-370 \mathrm{~m})$. Les formes sont au moins acceptables et, au mieux, très bonnes (tableau IX). Dans les sites sans risques majeurs de sécheresse, un second volet de provenances est à recommander : Pamuçak, Bakara, et Baspinar. Elles correspondent à des origines d'altitudes faibles à moyennes $(300-780 \mathrm{~m})$, les formes étant «très bonnes" à "exceptionnelles».

Enfin, la provenance Alexandropolis mérite d'être mieux étudiée en raison de sa bonne résistance au gel et à la sécheresse et des croissances observées sur les 2 sites expérimentaux.

\section{REMERCIEMENTS}

Cette étude a pu être réalisée grâce au financement de la Région Provence Alpes Côte d'Azur et de la Communauté économique européenne dans le cadre d'un programme intégré méditerranéen. 


\section{RÉFÉRENCES}

Allemand $P$, Auge $P$, Birot $Y$, Ferrandes $P$ (1985) Premiers enseignements des effets du froid de janvier 1985 sur les espèces forestières étudiées en arboretum et en plantations comparatives de provenances en région méditerranéenne française. For Méditer VII, 1, 85-90

Allemand $P$ (1989) Espèces exotiques utilisables pour la reconstitution du couvert végétal en région méditerranéenne. Bilan des arboretums forestiers d'élimination. INRA, Paris

Alptekin U (1990) Halep çami (Pinus halepensis Mill), in Türkiye agaçlandirma çalismalarindaki yeri ve dogal yayilisina ait bazi tesbitler (Quelques réflexions sur l'aire naturelle du pin d'Alep en Turquie et sur son importance dans les reboisements). Univ For, Istanbul, $12 \mathrm{p}$

Arbez M (1974) Répartition, écologie et variabilité de Pinus brutia en Turquie. Inf Ressources Génét For $3, \mathrm{FAO}, 24-37$

Bellefontaine R, Raggabi M (1977) Contribution à l'étude des pins de la section halepensis (P brutia, $P$ eldarica, $P$ halepensis) au Maroc: considérations générales et essai international de provenances. Ann Rech For Maroc 17, 191-234

Calamassi R, Falusi M, Tocci A (1980) Variazione geografica e resistenza a stress idrici in semi di Pinus halepensis Mill, Pinus brutia Ten Pinus eldarica Medw. Ann Ist Sper Selv Arezzo, XI, 195-230

Debazac EF (1964) Manuel des Conifères. EN. GREF, Nancy

Debazac E, Tomassone R (1965) Contribution à une étude comparée des pins méditerranéens de la section halepensis. Ann Sci For $22,216-254$
Demolin G (1969) Comportement des adultes de Thaumetopoea pityocampa Schiff. Dispersion spatiale, importance écologique. Ann Sci For 26, 81-102

Eccher A (1975) Proposition de méthodes standard pour l'étude comparée de provenances des pins méditerranéens de la section halepensis. Protocole FAO/SCM/CRFM/4bis, $14 \mathrm{p}$

Eccher A, Fusaro E, Pelleri F (1987) Résultats de l'expérimentation italienne sur les principales provenances de pins de la section halepensis dix ans après la plantation. For Méditerr, IX, 1, 5-14

Nahal I (1962) Le pin d'Alep (Pinus halepensis Mill). Étude taxinomique, phytogéographique, écologique et sylvicole. Ann EC Nat Eaux For, XIX, $208 \mathrm{p}$

Nouals D, Boisseau B (1991) Le pin brutia en France continentale. CEMAGREF, Aix-enProvence, $86 \mathrm{p}$

Panetsos KP (1986) Genetics and breeding in the group halepensis. In: Le pin d'Alep et le pin brutia dans la sylviculture méditerranéenne. Séries Études CIHEAM, 99-108

Pelizzo A, Tocci A (1978) Indagini preliminari su semi e semenzali di Pinus halepensis e Pinus brutia-eldarica. Ann Ist Sper Selv Arezzo, IX, 111-130

Quezel P (1980) Biogéographie et écologie des Conifères sur le pourtour méditerranéen. $A C$ tual Ecol For, 205-255

Quezel P, Barbero M (1985) Carte de la végétation potentielle de la région méditerranéenne. Feuille $n^{\circ} 1$ : Méditerranée orientale. CNRS, Paris, $65 p$

Weinstein A (1989) Provenance evaluation of Pinus halepensis, $P$ brutia and $P$ eldarica in Israel. For Ecol Manage 26, 215-225 